\title{
External Validation of Risk Prediction Scores for Invasive Candidiasis in a Medical/Surgical Intensive Care Unit: An Observational Study
}

\author{
Armin Ahmed, Arvind Kumar Baronia, Afzal Azim, Rungmei S. K. Marak¹, Reema Yadav¹, Preeti Sharma', Mohan Gurjar, Banani Poddar, Ratender Kumar Singh \\ Departments of Critical Care Medicine and 'Microbiology, Sanjay Gandhi Postgraduate Institute of Medical Sciences, Lucknow, Uttar Pradesh, India
}

\section{Abstract}

Background: The aim of this study was to conduct external validation of risk prediction scores for invasive candidiasis. Methods: We conducted a prospective observational study in a 12-bedded adult medical/surgical Intensive Care Unit (ICU) to evaluate Candida score $>3$, colonization index $(\mathrm{CI})>0.5$, corrected CI $>0.4(\mathrm{CCI})$, and Ostrosky's clinical prediction rule (CPR). Patients' characteristics and risk factors for invasive candidiasis were noted. Patients were divided into two groups; invasive candidiasis and no-invasive candidiasis. Results: Of 198 patients, 17 developed invasive candidiasis. Discriminatory power (area under receiver operator curve [AUROC]) for Candida score, CI, CCI, and CPR were $0.66,0.67,0.63$, and 0.62 , respectively. A large number of patients in the no-invasive candidiasis group (114 out of 181) were exposed to antifungal agents during their stay in ICU. Subgroup analysis was carried out after excluding such patients from no-invasive candidiasis group. AUROC of Candida score, CI, CCI, and CPR were $0.7,0.7,0.65$, and 0.72 , respectively, and positive predictive values (PPVs) were in the range of $25 \%-47 \%$, along with negative predictive values (NPVs) in the range of $84 \%-96 \%$ in the subgroup analysis. Conclusion: Currently available risk prediction scores have good NPV but poor PPV. They are useful for selecting patients who are not likely to benefit from antifungal therapy.

Keywords: Candida score, clinical prediction rule, colonization index, corrected colonization index, invasive candidiasis

\section{INTRODUCTION}

Invasive candidiasis can manifest as candidemia, deep-seated candidiasis, or a combination of both. ${ }^{[1]}$ Candidemia is the most common form of invasive candidiasis, followed by intra-abdominal candidiasis. Early microbiological diagnosis of invasive candidiasis still remains a difficult issue. ${ }^{[2,3]}$ Delay in the initiation of appropriate antifungal therapy is associated with increased mortality and morbidity ${ }^{[4,5]}$ Early identification of invasive candidiasis is essential for appropriate management and includes identification of high-risk groups. Risk assessment for invasive candidiasis includes assessment of clinical risk factors and colonization assessment. Common risk factors include broad-spectrum antibiotic use, pancreatitis, abdominal surgery, severe sepsis, total parenteral nutrition (TPN), hemodialysis, steroids, immunosuppression, and diabetes mellitus. ${ }^{[6]}$

Insight into pathogenesis has shown that invasive candidiasis mostly occurs after endogenous colonization in the predisposed

\begin{tabular}{|l|l|}
\hline \multicolumn{2}{|c|}{ Access this article online } \\
\hline Quick Response Code: & Website: \\
\hline & www.ijccm.org \\
\hline & \\
\hline
\end{tabular}

host. There is generally a latent period of 7-10 days between exposure to risk factor and actual development of infection. ${ }^{[7]}$ This latent period is the time during which progressive colonization develops that may or may not convert into invasive disease. Exogenous infection can occur but is less frequent than endogenous infection.

Risk factors for invasive candidiasis have been grouped together to design risk prediction scores/rules by various researchers. ${ }^{[8]}$ Due to low prevalence of invasive candidiasis $(0.5 \%-10 \%)$ in most settings, the specificity of these rules remains in the range of $50 \%-80 \%$ and positive predictive value (PPV) between $1 \%$ and $30 \%$ in most studies. ${ }^{[3]}$

Address for correspondence: Dr. Afzal Azim, Department of Critical Care Medicine, Sanjay Gandhi Postgraduate Institute of Medical Sciences, Lucknow - 226014 , Uttar Pradesh, India. E-mail: draazim2002@gmail.com

This is an open access article distributed under the terms of the Creative Commons Attribution-NonCommercial-ShareAlike 3.0 License, which allows others to remix, tweak, and build upon the work non-commercially, as long as the author is credited and the new creations are licensed under the identical terms.

For reprints contact: reprints@medknow.com

How to cite this article: Ahmed A, Baronia AK, Azim A, Marak RS, Yadav R, Sharma P, et al. External validation of risk prediction scores for invasive candidiasis in a medical/surgical intensive care unit: An observational study. Indian J Crit Care Med 2017;21:514-20. 
Risk prediction scores/rules for invasive candidiasis have been classified as scores based on clinical risk factors (clinical prediction rules [CPRs]), scores based on microbiological parameters (Candida colonization), and scores combining both clinical and microbiological (Candida score). ${ }^{[8]}$ The current study was undertaken to validate the commonly used prediction scores namely Ostrosky's CPR, colonization index (CI), corrected CI (CCI), and Candida score in critically ill patients with invasive candidiasis. ${ }^{[9-11]}$

\section{Methods}

\section{Patient selection and data collection}

The study was conducted under Senior Research Associateship Program of Council of Scientific and Industrial Research, New Delhi. Two hundred consecutive patients admitted to the 12-bedded general purpose Intensive Care Unit (ICU) of the Department of Critical Care Medicine of a tertiary care center from July 2013 to November 2014 were studied prospectively. Our ICU is a referral center, and we get patients from other ICUs in the institute as well as other hospitals in the city and nearby area. The study was approved by the ethics committee of the institute.

\section{Population}

Patients with absolute neutrophil count $<0.5 \times 10^{9} / \mathrm{L}$ at admission or during their stay in ICU and/or patients with hematological malignancy or bone marrow transplantation were excluded. All patients with age $<18$ years and those who died within $48 \mathrm{~h}$ of ICU admission were also excluded.

\section{Surveillance sample collection}

Fungal surveillance samples (oral swabs, tracheal aspirates, urine, axillary swab, and rectal swab) were collected at admission, $3^{\text {rd }}$ day, and then weekly for 3 weeks. Other samples were sent from drain fluid, necrosectomy tissue, pus/wound discharge, and vascular catheters as per the standard protocol of the ICU. Paired blood cultures were sent at admission and then as per decision of the treating physician.

\section{Microbiological technique}

A loopful of specimen was taken with a sterile loop and streaked on Sabourad dextrose agar plate and inoculated at $37^{\circ} \mathrm{C}$. The cultures were observed at 24 and $48 \mathrm{~h}$ for growth of yeast colonies. The colonies were classified as scanty $\left(<10^{3} \mathrm{CFU}\right)$, moderate $\left(10^{4} \mathrm{CFU}\right)$, heavy $\left(>10^{5} \mathrm{CFU}\right)$. Processing of blood culture was done using automated BACTEC system (Becton Dickinson Diagnostic Instrument system). Samples showing growth of Candida isolates were subjected to phenotypic species identification through germ tube testing, sugar assimilation, chrome agar, and tetrazolium reduction medium. Isolates from blood were sent for Matrix-Assisted Laser Desorption Ionization (MALDI)-Time of Flight; Bruker Daltonik MALDI Biotyper analysis.

\section{Data collection}

Patients' demographic characters, length of ICU stay, survival at 28 days and at discharge, and risk factors for invasive candidiasis were noted. Patients were followed until discharge from ICU or death. Patients were classified as medical or surgical according to the reason for admission. Surgical patients were those who were admitted for the postoperative management of an elective or urgent procedure. Medical patients who underwent surgery during their ICU stay were also classified as surgical. Acute Physiology And Chronic Health Evaluation Score (APACHE II) and Sequential Organ FAilure (SOFA) scores were recorded at admission. ${ }^{[12,13]}$ Use of steroids was defined as corticosteroid use of daily dose of prednisolone $20 \mathrm{mg}$ or equivalent for at least 2 weeks. Parenteral nutrition was defined as use of partial parental nutrition and/or TPN for at least $24 \mathrm{~h}$ or more. Invasive candidiasis was defined as per the consensus definition of European Organization for Research and Treatment of Cancer/Invasive Fungal Infections Cooperative Group/National Institute of Allergy and Infectious Diseases Mycoses Study Group Consensus Group. ${ }^{[14]}$ Only patients with proven invasive candidiasis as demonstrated by positive culture from blood (candidemia)/sterile body site collected surgically or during placement of percutaneous catheter (deep-seated candidiasis) were defined as cases.

\section{Risk prediction score/models}

The performance characteristics of four previously derived risk prediction models were evaluated in the study cohort. All scores were calculated at admission, $3^{\text {rd }}$ day, and then weekly till 3 weeks. Candida score was used as described by León et al. ${ }^{[9]}$ The components of Candida score were severe sepsis, parenteral nutrition, multifocal colonization, and surgery. For calculating Candida score, each component was given 1 point, except for severe sepsis which was given 2 points. The points were summed to calculate Candida score.

Severe sepsis was defined as sepsis associated with organ dysfunction, hypoperfusion, or hypotension. ${ }^{[15]}$ The manifestations of hypoperfusion included, but were not limited to, lactic acidosis, oliguria, or acute alteration in mental status. Colonization was defined as the presence of Candida species in samples obtained from distinct body sites, the oropharynx, urine, or tracheal aspirates. Colonization was considered unifocal when Candida species was isolated from one focus and multifocal when Candida species were simultaneously isolated from various noncontiguous foci even if two different Candida species were isolated. A score $>3$ was taken as positive.

CI and CCI were calculated as described by Pittet et al..$^{[10]}$ CI was calculated as nonblood distinct body sites colonized by Candida divided by total number of sites tested. CCI was calculated by multiplying CI to ratio of heavy growth distinct body sites on total number of distinct body sites positive for Candida. $\mathrm{CI}$ of $>0.5$ and $\mathrm{CCI}>0.4$ were taken as positive.

Clinical prediction score (CPR) was used as described by Ostrosky-Zeichner et al. ${ }^{[11]}$ The rule included intensive care admission for $72 \mathrm{~h}$ with mechanical ventilation for at least $48 \mathrm{~h}$ with antibiotic use for 3 days with central line use for 3 days with at least any one of the following criteria; any surgery 
(day-7 to day 0), immunosuppressive use (day-7 to day 0 ), pancreatitis (day-7 to day 0 ), TPN (day 1 to day 3 ), any dialysis (day 1 to day 3 ), steroid use (day -7 to day 0 ).

During the study period, all process of patient management was determined by the treating physician.

\section{Statistical analysis}

Patients were divided into two groups; invasive candidiasis group and no-invasive candidiasis group. Statistical analysis was done using SPSS 17 version (IBM). All the categorical variables were expressed as frequencies and percentages, while the continuous variables were expressed as mean with standard deviation when the distribution was Gaussian while medians with interquartile range (IQR 25-75) were used when distribution was nonparametric. Chi-square test was used for discrete variables while Mann-Whitney test was used for continuous variables when distribution was nonparametric and Student's $t$-test was used for parametric distribution. A $P<0.05$ was taken as statistically significant. Sensitivity, specificity, PPV and negative predictive value (NPV) were calculated from online software MedCalc (www.medcalc.org).

\section{RESULTS}

During the study period, there were 305 ICU admissions, of which 200 patients qualified the inclusion criteria [Figure 1]. Two patients were excluded from analysis as one patient developed invasive candidiasis (drain fluid positive) after being discharged to the ward and another developed invasive candidiasis (blood culture positive) on day 39 of ICU admission (colonization data unavailable).

One hundred ninety-eight patients were included for analysis [Table 1]. There were 124 males and 74 females. Mean age of the cohort was $45 \pm 16.7$ years. There were 165 medical and 33 surgical patients. At admission, median APACHE II

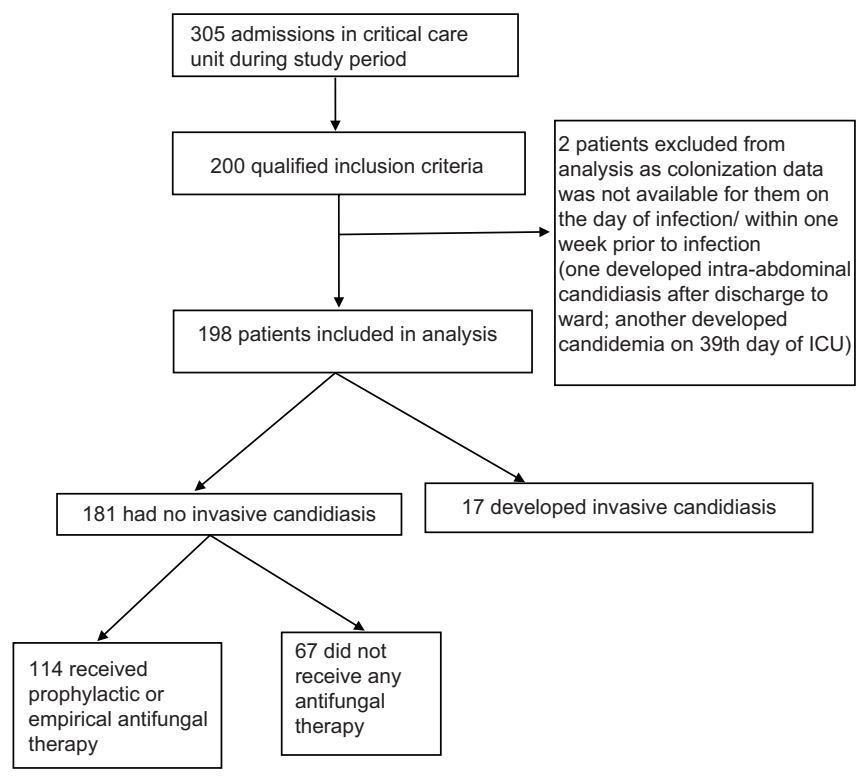

Figure 1: Diagram showing recruitment of patients and SOFA scores were 17 and 10, respectively. Median length of ICU stay was 12.5 (IQR 7-29) days and hospital stay was 23 (15-46) days. Survivals at 28 days and at discharge from ICU were $56.5 \%$ and $51 \%$, respectively.

Median time to development of invasive candidiasis from day of hospitalization was 21 (IQR10-28) days. The rate of invasive candidiasis was $4.5 / 1000$ patient days (17/3763 days). There was no significant difference in the age, gender, APACHE II score, and SOFA score between the two groups. Invasive candidiasis patients had increased duration of ICU stay, hospital stay, mechanical ventilation, and septic shock. Survival was not different among the two groups [Table 2].

\section{Risk factors}

Among the various risk factors studied, surgery, pancreatitis, number of invasive lines, and duration of pre-ICU hospitalization were significantly different in the two groups on univariate analysis [Table 3]. Candida score, CI, and patients qualifying CPR were significantly higher in invasive candidiasis group ( $P=0.017,0.044$, and 0.011 , respectively). Mean CCI was higher in invasive candidiasis group $(0.37 \pm 0.26)$ as compared in no-invasive candidiasis group $(0.25 \pm 0.23)$, but the value did not reach statistical significance $(P=0.096)$.

\section{Candida infections}

Invasive candidiasis developed in 17 patients. Three patients had candidemia as well as deep-seated candidiasis (necrosum/drain fluid positive), one patient had isolated deep-seated candidiasis (drain fluid positive), and 13 patients had isolated candidemia.

\section{Table 1: Demographic data}

\begin{tabular}{lc}
\hline Variable & $\begin{array}{c}\text { Total population } \\
(\boldsymbol{n}=198), \boldsymbol{n}(\%)\end{array}$ \\
\hline Age & \\
Mean \pm SD & $45 \pm 16.7$ \\
Median & $45.5(30-60)$ \\
Male & $124(63)$ \\
Female & $74(37)$ \\
Medical & $165(83)$ \\
Surgical & $33(17)$ \\
SOFA at ICU admission & $10(7-13)$ \\
APACHE II at ICU admission & $17(12-22)$ \\
Diagnosis & \\
Neurological & $25(12.5)$ \\
Cardiovascular & $13(6.5)$ \\
Respiratory & $32(16)$ \\
Abdominal & $62(31)$ \\
Renal & $14(7)$ \\
Tropical (dengue, malaria, typhoid, & $24(12)$ \\
tuberculosis) & \\
Others (poisoning, trauma, autoimmune, & $28(14)$ \\
malignancy) & \\
\hline SD: Standard deviation; SOFA: Sepsis related organ failure assessment \\
score; ICU: Intensive Care Unit; APACHE II: Acute Physiology and \\
Chronic Health Evaluation II
\end{tabular}




\begin{tabular}{lccc}
\hline Table 2: Outcome variables & & \\
\hline Variable & $\begin{array}{c}\text { Total population } \\
(\boldsymbol{n = 1 9 8 )}\end{array}$ & $\begin{array}{c}\text { Invasive candidiasis } \\
(\boldsymbol{n = 1 7 )}\end{array}$ & $\begin{array}{c}\text { No invasive } \\
\text { candidiasis }(\boldsymbol{n}=\mathbf{1 8 1})\end{array}$ \\
\hline Days of hospital stay, median (IQR) & $22.5(15-46)$ & $46(33-58)$ & $22(15-41)$ \\
Duration of mechanical ventilation in days, median (IQR) & $12(6-25)$ & $32(14-50)$ & $11(6-24)$ \\
Duration of septic shock in days, median (IQR) & $5(2-9.5)$ & $11(4.5-23)$ & 0.002 \\
Survival at 28 days & $112(56.5)$ & $11(65)$ & 0.001 \\
Survival at discharge from ICU & $102(51)$ & $9(53)$ & 0.020 \\
\hline
\end{tabular}

IQR: Interquartile range; ICU: Intensive Care Unit

\section{Table 3: Risk factors for Invasive candidiasis}

\begin{tabular}{|c|c|c|c|c|}
\hline Variable & $\begin{array}{l}\text { Total population } \\
\quad(n=198)\end{array}$ & $\begin{array}{l}\text { Invasive candidiasis } \\
\qquad(n=17)\end{array}$ & $\begin{array}{l}\text { No invasive candidiasis } \\
\qquad(n=181)\end{array}$ & $P$ \\
\hline Days of hospitalization before ICU admission, median (IQR) & $7(3.75-13)$ & $14(7-25.5)$ & $7(3-12)$ & 0.011 \\
\hline Surgery, $n(\%)$ & $33(17)$ & $8(47)$ & $25(14)$ & $<0.001$ \\
\hline Diabetes mellitus, $n(\%)$ & $41(20)$ & $2(11)$ & $39(21)$ & 0.341 \\
\hline Pancreatitis, $n(\%)$ & $28(14)$ & $7(41)$ & $22(12)$ & 0.001 \\
\hline Organ transplant, $n(\%)$ & $9(4.5)$ & 0 & $9(4.9)$ & 0.345 \\
\hline Total parenteral nutrition/partial parenteral nutrition, $n(\%)$ & $128(65)$ & $13(76)$ & $115(63.5)$ & 0.286 \\
\hline Number of invasive lines and catheters, median (IQR) & $4(3-4)$ & $5(3-6)$ & $4(3-4)$ & 0.013 \\
\hline Septic shock at admission, $n(\%)$ & $138(70)$ & $13(76)$ & $125(69)$ & 0.525 \\
\hline Use of mechanical ventilation, $n(\%)$ & $180(91)$ & $16(94)$ & $164(91)$ & 0.630 \\
\hline Use of RRT, $n(\%)$ & $97(49)$ & $8(52)$ & $89(48)$ & 0.868 \\
\hline Use of steroids, $n(\%)$ & $27(14)$ & $2(12)$ & $25(14)$ & 0.814 \\
\hline Any colonization (at admission or during ICU stay), $n(\%)$ & $189(95)$ & $16(94)$ & $173(95.5)$ & 0.782 \\
\hline Multifocal colonization (at admission or during ICU stay), $n(\%)$ & $160(81)$ & $16(94)$ & $144(79.5)$ & 0.145 \\
\hline Candida score, mean \pm SD & $3.03 \pm 0.94$ & $3.5 \pm 0.7$ & $2.9 \pm 0.94$ & 0.017 \\
\hline $\mathrm{CI}$, mean $\pm \mathrm{SD}$ & $0.45 \pm 0.29$ & $0.59 \pm 0.30$ & $0.44 \pm 0.29$ & 0.044 \\
\hline $\mathrm{CCI}$, mean $\pm \mathrm{SD}$ & $0.26 \pm 0.23$ & $0.37 \pm 0.26$ & $0.25 \pm 0.23$ & 0.096 \\
\hline CPR (frequency of qualified patients), $n(\%)$ & $53(27)$ & $9(53)$ & $44(24)$ & 0.011 \\
\hline
\end{tabular}

IQR: Interquartile range; SD: Standard deviation; ICU: Intensive Care Unit; RRT: Renal replacement therapy; CPR: Clinical prediction rule;

CI: Colonization index; CCI: Corrected colonization index

\section{Candida species}

Blood cultures of 16 patients were positive for Candida spp. Thirteen patients were positive at admission while three patients showed growth of Candida at $13^{\text {th }}, 15^{\text {th }}, 21^{\text {st }}$ day of ICU stay. Three patients were diagnosed as multispecies candidemia as they showed growth of more than one species from same blood culture bottle or sample collected within $72 \mathrm{~h}$. Candida tropicalis ( 7 isolates) was the most common species responsible for candidemia, followed by Candida parapsilosis (4 isolates). Other species were Candida glabrata (3 isolates), Candida auris (3 isolates), Candida albicans (2 isolates), and Candida rugosa (1 isolate).

Deep-seated candidiasis (intra-abdominal specimen/drain fluid positive for Candida) was found in four patients, out of which one had isolated deep-seated candidiasis while three had deep-seated candidiasis with candidemia. Species identification of intra-abdominal isolates was not done.

\section{Candida risk prediction models/scores}

Scores of the invasive candidiasis patients on the day or within 1 week before culture positivity were compared with admission scores of no-invasive candidiasis group. Candida score was the most sensitive (94.1\%) while CPR was the most specific (75.7\%). PPV and NPV ranged from $10 \%$ to $17 \%$ and $92 \%$ to $98 \%$, respectively [Table 4]. Area under curves for Candida score, CI, CCI, and CPR were $0.66,0.67,0.63$, and 0.62 , respectively [Figure 2].

A large number of patients in the no-invasive candidiasis group (114 out of 181) were exposed to antifungal agents during their stay in the ICU. Some of these patients might have developed invasive candidiasis if empirical/prophylactic antifungal therapy had not been used. Therefore, subgroup analysis was done after excluding the patients who were exposed to antifungal agents in the no-invasive candidiasis group $(n=67)$ [Table 5]. Discriminatory power of the scores improved with area under curves for Candida score, CI, CCI, and CPR were 0.70, 0.70, 0.65 , and 0.72 , respectively [Figure 3 ].

\section{Discussion}

In agreement with other studies, this study shows that invasive candidiasis is associated with increased days of hospitalization and morbidity. Risk prediction scores are helpful in early the identification of patients likely to develop invasive candidiasis. 


\section{Table 4: Performance of risk prediction models/scores $(n=198)$}

\begin{tabular}{lcccc}
\hline & Candida score & Cl & CCI & Ostrosky's CPR \\
\hline Sensitivity percentage $(95 \% \mathrm{CI})$ & $94.1(71.3-99.8)$ & $58.8(32.9-81.6)$ & $52.9(27.8-77.0)$ & $52.9(22.8-77.0)$ \\
Specificity percentage $(95 \% \mathrm{CI})$ & $24.3(18.3-21.2)$ & $50.7(42.8-57.8)$ & $66.9(59.5-73.7)$ & $75.7(68.8-81.8)$ \\
PPV percentage $(95 \% \mathrm{CI})$ & $10.5(6.1-16.4)$ & $10(4.9-17.6)$ & $13.0(6.1-23.3)$ & $17.0(8.1-29.8)$ \\
NPV percentage $(95 \% \mathrm{CI})$ & $97.8(88.2-99.9)$ & $92.9(85.8-97.0)$ & $93.80(88.2-97.3)$ & $94.5(89.4-97.6)$ \\
PLR $(95 \% \mathrm{CI})$ & $1.24(1.08-1.44)$ & $1.18(0.77-1.81)$ & $1.60(0.97-2.62)$ & $2.18(1.30-3.65)$ \\
NLR $(95 \% \mathrm{CI})$ & $0.24(0.04-1.65)$ & $0.82(0.46-1.47)$ & $0.71(0.42-1.18)$ & $0.54(0.31-0.97)$ \\
Area under curve $(95 \% \mathrm{CI})$ & $0.66(0.54-0.79)$ & $0.67(0.54-0.80)$ & $0.63(0.49-0.77)$ & $0.62(0.37-1.04)$ \\
\hline
\end{tabular}

Not applicable. PPV: Positive predictive value; NPV: Negative predictive value; PLR: Positive likelihood ratio; NLR: Negative likelihood ratio; 95\% CI: 95\% confidence interval; CPR: Clinical prediction rule; CI: Colonization index; CCI: Corrected colonization index

Table 5: Subgroup analysis; $n=84$ (performance of scores after excluding patients exposed to antifungal therapy in no-invasive candidiasis group)

\begin{tabular}{lcccc}
\hline & Candida score & CI & CCI & Ostrosky's CPR \\
\hline Sensitivity percentage $(95 \%$ CI) & $94.1(71.3-99.8)$ & $58.8(32.9-81.6)$ & $52.9(27.8-77.0)$ & $52.9(22.8-77.0)$ \\
Specificity percentage $(95 \%$ CI) & $40.3(28.5-53.0)$ & $56.7(44.0-68.8)$ & $77.6(65.8-86.9)$ & $85.0(74.3-92.6)$ \\
PPV percentage $(95 \%$ CI) & $28.6(17.3-42.2)$ & $25.64(13.0-42.1)$ & $37.5(18.8-59.4)$ & $47.4(24.5-71.1)$ \\
NPV percentage $(95 \%$ CI) & $96.4(81.6-99.9)$ & $84.4(70.5-93.5)$ & $86.7(75.4-94.0)$ & $87.7(77.2-94.5)$ \\
PLR (95\% CI) & $1.58(1.25-1.98)$ & $1.36(0.84-2.20)$ & $2.4(1.26-4.45)$ & $3.5(1.72-7.33)$ \\
NLR (95\% CI) & $0.15(0.02-1.00)$ & $0.73(0.40-1.33)$ & $0.61(0.36-1.02)$ & $0.55(0.33-0.92)$ \\
Area under curve $(95 \%$ CI) & $0.70(0.56-0.84)$ & $0.70(0.56-0.82)$ & $0.65(0.50-0.80)$ & $0.72(0.57-0.87)$ \\
\hline
\end{tabular}

Not applicable. PPV: Positive predictive value; NPV: Negative predictive value; PLR: Positive likelihood ratio; NLR: Negative likelihood ratio; CPR: Clinical prediction rule; CI: Colonization index; CCI: Corrected colonization index; 95\% CI: 95\% confidence interval

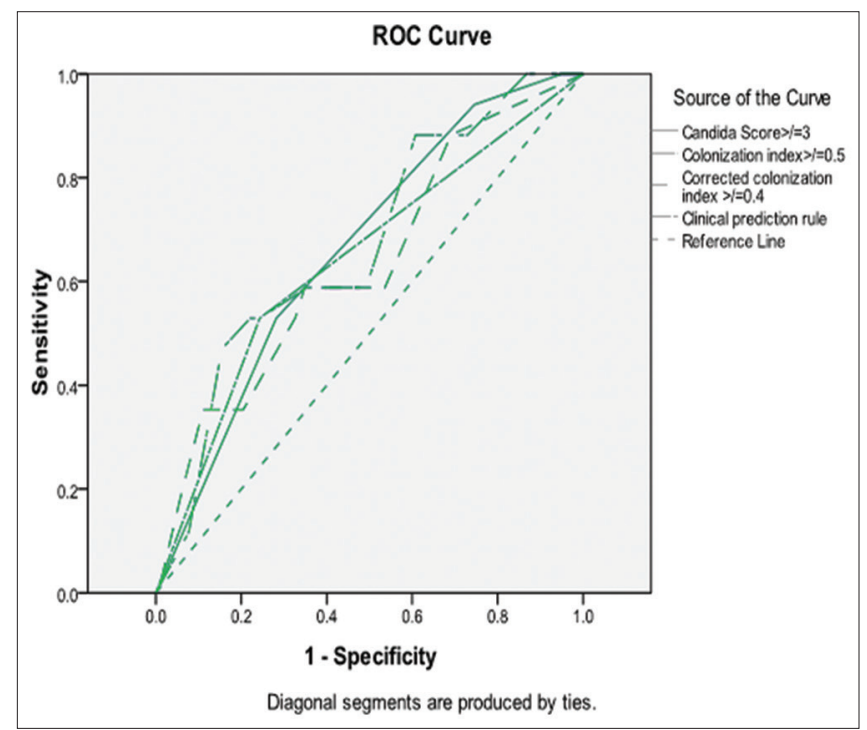

Figure 2: Receiver operator curve $(n=198)$ showing discriminatory power of four risk prediction scores

To the best of our knowledge, this is the first external validation study of risk prediction scores for invasive candidiasis done in a tropical country. Our findings should be interpreted taking into account the high rate $(8.5 \%)$ of invasive candidiasis and Candida colonization in the cohort. Ninety-five percent of our patients were colonized with Candida at admission or during their stay in ICU and $81 \%$ patients had multifocal colonization. Candida score and CI $>0.5$ had the best discriminatory power in our study population (area under receiver operator

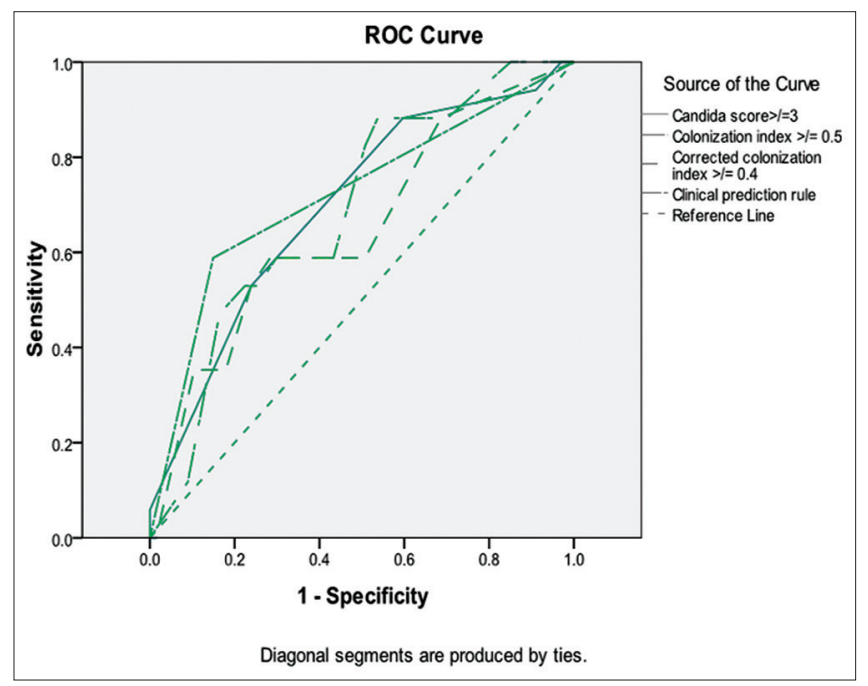

Figure 3: Receiver operator curve (subgroup analysis [ $n=84]$ ) showing discriminatory power of four risk prediction scores

curve [AUROC] 0.66 and 0.67 , respectively). However, after eliminating the patients who received prophylactic/empirical antifungal therapy from no-invasive candidiasis group, performance of Candida score $>3, \mathrm{CI}>0.5$, and CPR were comparable (AUROC 0.7, 0.7, 0.72, respectively).

\section{Comparison with other external validation studies in literature}

The external validation of Ostrosky's original and revised CPRs has been previously done by Playford et al ${ }^{[16]}$ The study was conducted in four major Australian ICUs and included 
615 critically ill patients, out of which 15 developed invasive candidiasis. CCI $>0.4$ showed the best discriminatory power. AUROC of CPR 1 (Ostrosky's old model), CPR 2 (Ostrosky's revised model), CI, and CCI was $0.63,0.66,0.74$, and 0.75 , respectively. Authors showed that addition of CI to CPRs improved the score performance.

Hermsen validated Ostrosky's CPR along with another rule given by Paphitou in a case-control study including 352 patients. ${ }^{[17]}$ The prediction rules showed low PPVs (4.1\%-5.4\%) and high NPVs (98.35\%-99\%) with AUROC of 0.649-0.705. Risk factors found to be significant in Ostrosky's and/or Paphitou's study such as pancreatitis, diabetes, and hemodialysis were not shown to be statistically significant in their study. New risk factors found statistically significant in their study cohort were abdominal surgery and length of pre-ICU stay. They developed their own risk prediction score named Nebraska Medical Centre score after including these new risk factors. Although our study was not designed to assess the risk factors due to small sample size, our findings are somewhat similar to Hermsen et al. study. We found that surgery and length of pre-ICU hospital stay were significantly different between invasive candidiasis and no-invasive candidiasis patients.

Hall et al. studied CI, CPR (original model with modification), and Candida score in 101 severe acute pancreatitis patients, out of which 18 had invasive candidiasis. ${ }^{[18]}$

CI $>0.5$ showed best performance with AUROC of 0.79 . Candida score and CPR (original model with modification) had an AUROC of 0.62 and 0.59, respectively. The cutoff used for Candida score in this study was $>3$ leading to a low sensitivity (23\%) and high specificity (85\%). We used a cutoff of $>3$ for Candida score as used by León et al. in their Candida score validation study on 1107 nonneutropenic ICU patients. ${ }^{[19]}$ Candida score had a sensitivity of $94.1 \%$ and specificity of $24.3 \%$ in our study.

Posteraro et al. compared the diagnostic accuracy of beta-d glucan (BDG) assay, Candida score, and CI in the single-center prospective study involving 95 patients. ${ }^{[20]}$ Fourteen patients developed invasive candidiasis. Beta-D-Glucan assay had an AUROC of 0.98, Candida score had AUROC of 0.80, while AUROC of CI was 0.63 . Authors recommended single-point BDG assay alone or in combination with Candida score may help in guiding antifungal therapy in high-risk patients.

Insight from the above discussion shows that discriminatory power of currently available risk scores for invasive candidiasis ranges between 0.6 and 0.8 depending on the study population and cutoffs used to define the score. By and large, they show good NPV and low PPV, thus helping to differentiate who are not likely to benefit from antifungal therapy.

\section{Can we improve risk prediction for invasive candidiasis?}

An insight into the various components of currently used models can help improve risk prediction for invasive candidiasis. Ostrosky's rule can be applied bedside without requiring any microbiological data. It has mandatory criteria as well as optional criteria. Duration of ICU stay (72 h) and duration of mechanical ventilation (48 h) are mandatory criteria along with antibiotic use (1-3 days) and central venous catheter use (1-3 days). These factors may hinder the general applicability of the rule as indication for admission to ICU may vary from country to country and even hospital to hospital in one country. Even threshold for starting mechanical ventilation may vary from center to center.

Along with mandatory criteria, the score requires fulfillment of any one criteria out of the following; any surgery, immunosuppressive use, pancreatitis, TPN, any dialysis, steroid use. Among the criteria which are optional, some patients may qualify the score due to one factor while others may qualify due to two or more factors. A patient qualifying due to more than one factor is at higher risk than the one qualifying due to one factor, but this discrimination is not possible in the rule. If these factors are corrected in the future studies, Ostrosky's risk prediction rule can become an important bedside tool as it is readily applicable and does not require any waiting for microbiological results. We suggest duration of hospitalization rather than duration of ICU stay or mechanical ventilation as mandatory criteria for improvement of Ostrosky's risk prediction rule.

Candida score has four components; severe sepsis, TPN, surgery, and multifocal colonization. The definition of severe sepsis remains reevaluation according to the recent consensus guidelines. Besides this, our knowledge of nutritional support during $1^{\text {st }}$ week of critical illness is still evolving. Use of partial parenteral nutrition is more common than TPN. It is unclear whether it is one component of the TPN or it is the whole TPN which predisposes for Candida infection. Candida colonization is a well-proven risk factor for invasive candidiasis. Candida score uses multifocal colonization as a component to calculate the score. However, Lau et al. in a large multicenter study on 6015 nonneutropenic critically ill patients showed that colonization of two or more sites and heavy colonization of single site are independent risk factors for invasive candidiasis. ${ }^{[21]}$ The risk of infection was highest with urinary colonization in the study. We suggest more detailed evaluation of colonization such as type and number of sites and intensity to improve performance of Candida score.

CI and CCI given by Pittet et al. were generated on surgical population. ${ }^{[10]}$ They do not include clinical risk factors. The number of sites that should be studied for colonization surveillance and frequency of sample collection remain unanswered questions. Use of these indices is limited due to cost and increased burden on microbiology laboratory.

In our opinion, risk prediction for invasive candidiasis can be done in two-step approach. First step should include assessment of clinical risk factor. Patient's qualifying for clinical risk factors criteria should be subjected to microbiological surveillance by studying the intensity of colonization with or without biomarkers such as BDG assay at regular interval. Prophylactic antifungal should be started 
once the patient crosses a particular threshold for colonization or shows positive BDG assay.

\section{Limitations}

Our study is limited in its single-center design and small sample size. We collected colonization data only for first 21 days of ICU stay due to limited resources. Another weakness of our study was selection bias toward more serious sepsis patients because septic shock at admission was present in $69.5 \%$ patients and $90 \%$ patients required mechanical ventilation during their stay in ICU. This could be because our ICU is a referral center and we get patients from other ICUs in the institute as well as hospitals in the nearby area.

\section{Conclusion}

Invasive candidiasis is a serious problem in critically ill patients, leading to increased morbidity and economic burden. Risk prediction scores for invasive candidiasis are important for judicious use of antifungal need therapy. However, performance of these rules varies depending upon the study population and cutoffs used to define a positive case. Currently available rules can be best used to identify patients who are not likely to benefit from antifungal therapy.

\section{Acknowledgments}

We are in debt to Mr. Mandal and Dr. Ranjan for their valuable inputs in data analysis.

\section{Financial support and sponsorship}

Nil.

\section{Conflicts of interest}

There are no conflicts of interest.

\section{References}

1. Brusselaers N, Blot S, Vogelaers D. Deep-seated Candida infections in the Intensive Care Unit. Neth J Crit Care 2011;15:184-90.

2. Clancy CJ, Nguyen MH. Finding the "missing 50\%" of invasive candidiasis: How nonculture diagnostics will improve understanding of disease spectrum and transform patient care. Clin Infect Dis 2013;56:1284-92.

3. Kullberg BJ, Arendrup MC. Invasive candidiasis. N Engl J Med 2015;373:1445-56.

4. Morrell M, Fraser VJ, Kollef MH. Delaying the empiric treatment of Candida bloodstream infection until positive blood culture results are obtained: A potential risk factor for hospital mortality. Antimicrob Agents Chemother 2005;49:3640-5.

5. Garey KW, Rege M, Pai MP, Mingo DE, Suda KJ, Turpin RS, et al. Time to initiation of fluconazole therapy impacts mortality in patients with candidemia: A multi-institutional study. Clin Infect Dis 2006;43:25-31.

6. Muskett H, Shahin J, Eyres G, Harvey S, Rowan K, Harrison D, et al.
Risk factors for invasive fungal disease in critically ill adult patients: A systematic review. Crit Care 2011;15:R287.

7. Eggimann P, Pittet D. Candida colonization index and subsequent infection in critically ill surgical patients: 20 years later. Intensive Care Med 2014;40:1429-48.

8. Ahmed A, Azim A, Baronia AK, Marak KR, Gurjar M. Risk prediction for invasive candidiasis. Indian J Crit Care Med 2014;18:682-8.

9. León C, Ruiz-Santana S, Saavedra P, Almirante B, Nolla-Salas J, Alvarez-Lerma F, et al. A bedside scoring system ("Candida score") for early antifungal treatment in nonneutropenic critically ill patients with Candida colonization. Crit Care Med 2006;34:730-7.

10. Pittet D, Monod M, Suter PM, Frenk E, Auckenthaler R. Candida colonization and subsequent infections in critically ill surgical patients. Ann Surg 1994;220:751-8.

11. Ostrosky-Zeichner L, Pappas PG, Shoham S, Reboli A, Barron MA, Sims C, et al. Improvement of a clinical prediction rule for clinical trials on prophylaxis for invasive candidiasis in the Intensive Care Unit. Mycoses 2011;54:46-51.

12. Knaus WA, Draper EA, Wagner DP, Zimmerman JE. APACHE II: A severity of disease classification system. Crit Care Med 1985;13:818-29.

13. Vincent JL, Moreno R, Takala J, Willatts S, De Mendonça A, Bruining H, et al. The SOFA (Sepsis-related Organ Failure Assessment) score to describe organ dysfunction/failure. On behalf of the Working Group on Sepsis-Related Problems of the European Society of Intensive Care Medicine. Intensive Care Med 1996;22:707-10.

14. De Pauw B, Walsh TJ, Donnelly JP, Stevens DA, Edwards JE, Calandra T, et al. Revised definitions of invasive fungal disease from the European Organization for Research and Treatment of Cancer/Invasive Fungal Infections Cooperative Group and the National Institute of Allergy and Infectious Diseases Mycoses Study Group (EORTC/MSG) Consensus Group. Clin Infect Dis 2008;46:1813-21.

15. Levy MM, Fink MP, Marshall JC, Abraham E, Angus D, Cook D, et al. $2001 \mathrm{SCCM} / \mathrm{ESICM} / \mathrm{ACCP} / \mathrm{ATS} / \mathrm{SIS}$ international sepsis definitions conference. Intensive Care Med 2003;29:530-8.

16. Playford EG, Lipman J, Kabir M, McBryde ES, Nimmo GR, Lau A, et al. Assessment of clinical risk predictive rules for invasive candidiasis in a prospective multicentre cohort of ICU patients. Intensive Care Med 2009;35:2141-5.

17. Hermsen ED, Zapapas MK, Maiefski M, Rupp ME, Freifeld AG, Kalil AC, et al. Validation and comparison of clinical prediction rules for invasive candidiasis in Intensive Care Unit patients: A matched case-control study. Crit Care 2011;15:R198.

18. Hall AM, Poole LA, Renton B, Wozniak A, Fisher M, Neal T, et al. Prediction of invasive candidal infection in critically ill patients with severe acute pancreatitis. Crit Care 2013;17:R49.

19. León C, Ruiz-Santana S, Saavedra P, Galván B, Blanco A, Castro C, et al. Usefulness of the "Candida score" for discriminating between Candida colonization and invasive candidiasis in non-neutropenic critically ill patients: A prospective multicenter study. Crit Care Med 2009;37:1624-33

20. Posteraro B, De Pascale G, Tumbarello M, Torelli R, Pennisi MA, Bello $\mathrm{G}$, et al. Early diagnosis of candidemia in Intensive Care Unit patients with sepsis: A prospective comparison of $(1 \rightarrow 3)-\beta$-D-glucan assay, Candida score, and colonization index. Crit Care 2011;15:R249.

21. Lau AF, Kabir M, Chen SC, Playford EG, Marriott DJ, Jones M, et al. Candida colonization as a risk marker for invasive candidiasis in mixed medical-surgical Intensive Care Units: Development and evaluation of a simple, standard protocol. J Clin Microbiol 2015;53:1324-30. 